\title{
Conversational Semantic Role Labeling
}

\author{
Kun $\mathrm{Xu}^{1}$, Han $\mathrm{Wu}^{2}$, Linfeng Song ${ }^{1}$, Haisong Zhang ${ }^{1}$, Linqi Song ${ }^{2}$, and Dong $\mathrm{Yu}^{1}$ \\ ${ }^{1}$ Tencent AI Lab \\ ${ }^{2}$ City University of Hong Kong
}

\begin{abstract}
Semantic role labeling (SRL) aims to extract the arguments for each predicate in an input sentence. Traditional SRL can fail to analyze dialogues because it only works on every single sentence, while ellipsis and anaphora frequently occur in dialogues. To address this problem, we propose the conversational SRL task, where an argument can be the dialogue participants, a phrase in the dialogue history or the current sentence. As the existing SRL datasets are in the sentence level, we manually annotate semantic roles for 3,000 chit-chat dialogues $(27,198$ sentences) to boost the research in this direction. Experiments show that while traditional SRL systems (even with the help of coreference resolution or rewriting) perform poorly for analyzing dialogues, modeling dialogue histories and participants greatly helps the performance, indicating that adapting SRL to conversations is very promising for universal dialogue understanding. Our initial study by applying CSRL to two mainstream conversational tasks, dialogue response generation and dialogue context rewriting, also confirms the usefulness of CSRL.
\end{abstract}

\section{INTRODUCTION}

Recent years have witnessed increasing attentions of conversation-based tasks, such as dialogue response generation [1] -[5], task-oriented dialogue modeling $[6]-[8]$ and conversational question answering [9], [10]. As a central problem, ellipsis and anaphora frequently occur in human conversations, creating additional challenges for dialogue understanding. Specifically, ellipsis corresponds to the situations when a phrase or a clause that has been mentioned in the previous context is omitted for simplicity, and anaphora happens when a mention is replaced by a pronoun to avoid repetition. In addition to English, previous work [11] has shown that both phenomena can be much severer for pro-drop languages, including Japanese, Chinese and Korean.

One potential solution is to utilize SRL, a standard tool for semantic analysis inside most traditional NLU toolboxes [12], [13], to extract valuable information for the end task. Figure 1 shows a 3 -turn dialogue example in Chinese with SRL annotations, where relations like “雪宝 (Olaf)" being the "A1" argument of the predicate “喜欢 (like)" in the second turn clearly indicate the core subject-predicate-object relations (also fine-grained sentiment expressed by “喜欢 (like)"). However, traditional SRL can only work on every single sentence, thereby they may fail to capture the critical information across turns (utterances) for these conversational tasks. For instance, several semantic roles, such as "A0" and "AM-TMP" for the predicate "看了 (see)" in the second sentence, can be missed by traditional SRL, because these arguments are dropped from the sentence. Besides, it can be challenging to predict the relation between “雪宝 (Olaf)" with the predicate “喜欢 (like)" in the third turn, because that involves combining SRL ("它 (it)" being "A1" of “喜欢 (like)") with coreference resolution (“它 (it)” referring “雪宝 (Olaf)”).

To address this, previous approaches [14], [15] first perform sentence rewriting before conducting standard SRL, where sentence rewriting recovers dropped components and pronouns of coreference by copying from dialogue histories. For example, in Figure 11, the second turn can be rewritten as “我(I) 最近(recently) 着了(see) 冰雪奇缘2 (Frozen II), 我(I) 超

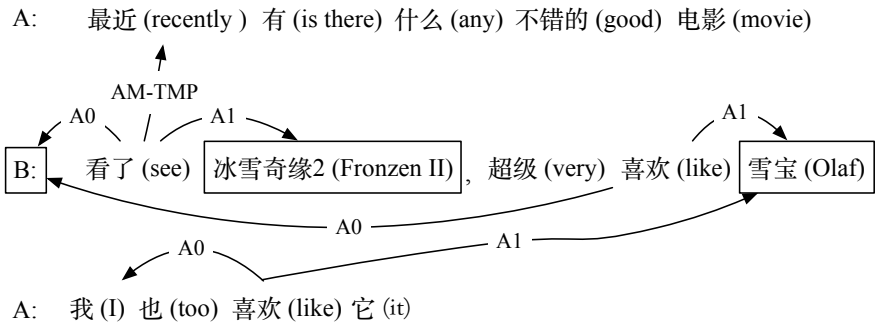

Fig. 1: A conversational SRL example. It covers all the core relations, while traditional SRL can only cover the relations indicated by black arrows. One slashed line indicates a coreference relation.

级(very) 喜欢(like) 雪宝(Olaf)", where recovered components are with underlines. Based on the rewritten sentence, missing cross-sentence information is supposed to be captured by standard SRL. However, the performance of the state-of-theart rewriting models are still far from satisfactory for out-ofdomain scenarios. This is probably because sentence rewriting is quite "subjective", as annotators usually have different ideas on the ideal rewriting outputs, resulting in high variance for the annotations.

In this paper, we introduce a new task, namely conversational semantic role labeling (CSRL), to directly model the predicate-argument structures across the entire conversation instead of an individual sentence. Our motivation behind proposing the task comes from one observation: while ellipsis and coreference frequently happen in each dialogue utterance, most dropped or referred components have been mentioned in the dialogue history, and very little information is missing from the whole dialogue. Following this observation, when analyzing a predicate in the latest dialogue turn, a CSRL model needs to consider not only the current turn but also its previous history sentences to search for potential arguments. Taking Figure 1 as an example, a system has to jointly consider the first two turns when analyzing the second turn (by speaker B), in order to capture all semantic roles. Compared with standard SRL that needs sentence rewriting or coreference resolution as 
a preprocessing step for analyzing dialogues, CSRL directly processes dialogues and can avoid error propagation.

There has been no publicly available data that contains the predicate-argument (PA) annotations beyond the sentence level. Therefore, as the first step to conversational SRL, we manually annotated DuConv [4], a popular dataset of Chinese chit-chat dialogues, with conversation-level semantic roles. Our corpus contains 3,000 dialogue sessions, including 27,198 utterances, 33, 673 predicates and their arguments. Besides, we respectively annotate 200 and 300 dialogue sessions from the corpus by [16] and an in-house dataset as out-ofdomain test sets to verify model robustness.

Experimental results demonstrate that our CSRL parser significantly outperforms the baselines trained on the standard CoNLL-2012 benchmark [17], though the standard benchmark contains four times more training instances than ours (roughly $117,089$ vs. 27,198$)$.

In addition, we make initial attempts that apply our CSRL parser on two dialogue downstream tasks, i.e., dialogue rewriting and dialogue generation. In particular, we first utilize our CSRL parser trained on our annotated dataset to extract PA structures, before incorporating this information into rewriting and generation baselines. Experiments on several benchmarks show that CSRL can significantly improve the state-of-the-art performances for both tasks.

In summary, our contributions are:

1) We propose a new task (i.e., CSRL) for modeling the PA structures in multi-turn dialogues. For this purpose, we collect an annotated dataset of high quality and two outof-domain datasets for robust testing, all of which can benefit future related research 1

2) We conduct detailed analysis to show that our model trained with our CSRL data largely outperforms the baselines trained on standard SRL data, and the improvements are consistent across different domains. Experimental results on out-of-domain datasets also demonstrate the robustness of our model for domain transfer.

3) Experimental results on the rewriting and dialogue generation datasets show that our CSRL parser could significantly improve existing baseline models by incorporating the predicate-argument information.

\section{RELATED WORK}

Semantic role labeling beyond sentence level. Until now, SRL is typically considered as a sentence-internal task, and the major SRL benchmarks [17], [18] only contain sentencelevel annotations. A partial reason can be that previous manual annotation projects choose to label isolated sentences to avoid wasting expensive human resources on rare long-range cases [19]. A few later efforts [19]-[21] propose discourse-based implicit argument linking as a remedy for long-range situations. Specifically, they introduce an implicit argument "null" in the target sentence for a cross-sentence argument, linking "null" to the argument. Conversely, we directly annotate the cross-sentence arguments for each predicate. These efforts only provide tens or hundreds of labeled instances while we

${ }^{1}$ https://github.com/syxu828/CSRL_dataset annotated more than $27 \mathrm{~K}$ sentences, enabling the training and evaluation for state-of-the-art neural models.

Dialogue understanding. It is essential to extract user intent for building successful dialogue systems. For taskoriented scenarios [22], the goal of dialogue understanding is definite, i.e., identifying the user intent (e.g. booking flight) and its corresponding semantic slots (e.g. from_city and to_city). In contrast, people lack consensus on what information can be helpful for modeling chit-chat dialogues, adding extra difficulties for building intelligent chatbots. Most previous efforts [23]-[29] present the surface string of dialogue histories by a neural encoder as the understanding results. They suffer from severe problems, such as being uninterpretable and generating trivial responses, like "I do not know". For the first time, we propose conversational semantic roles as generalpurpose representations for understanding chit-chat dialogues. The conversational semantic roles are symbolic, alleviating the uninterpretable problem. Comparatively, our predicates and arguments in chit-chat dialogues are reminiscent of the intent and slots for task-oriented dialogues.

A few recent efforts [30], [31] adopt topic modeling [32] for general dialogue understanding, where keywords are extracted from each dialogue utterance as the topic representation for that turn. Similar to conversational SRL, topic modeling also provides symbolic and interpretable features. Here we take one step further by modeling structures of predicates and arguments that can capture more complex information than individual words.

\section{CONVERSATIONAL SRL DATASET}

We annotate the predicate-argument structures on three datasets, including two public dialogue datasets: DuConv [4] and PersonalDialog [16], and one in-house dataset (referred as NewsDailog), each having a very different domain and style from the others. Following standard SRL, we adopt the semantic roles in PropBank to label the relationships between arguments. In this section, we first introduce the datasets (Section III-A) and the semantic roles based on PropBank (Section III-B), before showing our annotation details (Section III-C).

\section{A. Dialogue Datasets}

a) DuConv: It is a publicly available knowledge-driven dialogue dataset, focusing on the domain of movies and stars. It consists of $30 \mathrm{k}$ dialogues with $270 \mathrm{k}$ dialogue turns and provides a knowledge graph $(\mathrm{KG})$ in the domain of movies and celebrities. Each dialogue is collected by asking two crowdsourced annotators to conduct a multi-turn KG grounded conversation focusing on given entities, where one plays the role of the conversation leader and the other one acts as the conversation follower.

b) PersonalDialog: In contrast to DuConv, which involves only the domain of movies and stars, PersonalDialog includes more than 250 domains. It is collected by crawling Weibq ${ }^{2}$ posts and comments. Specifically, when a user posts

\footnotetext{
${ }^{2} \mathrm{~A}$ Chinese version of Twitter, http://www.weibo.com
} 


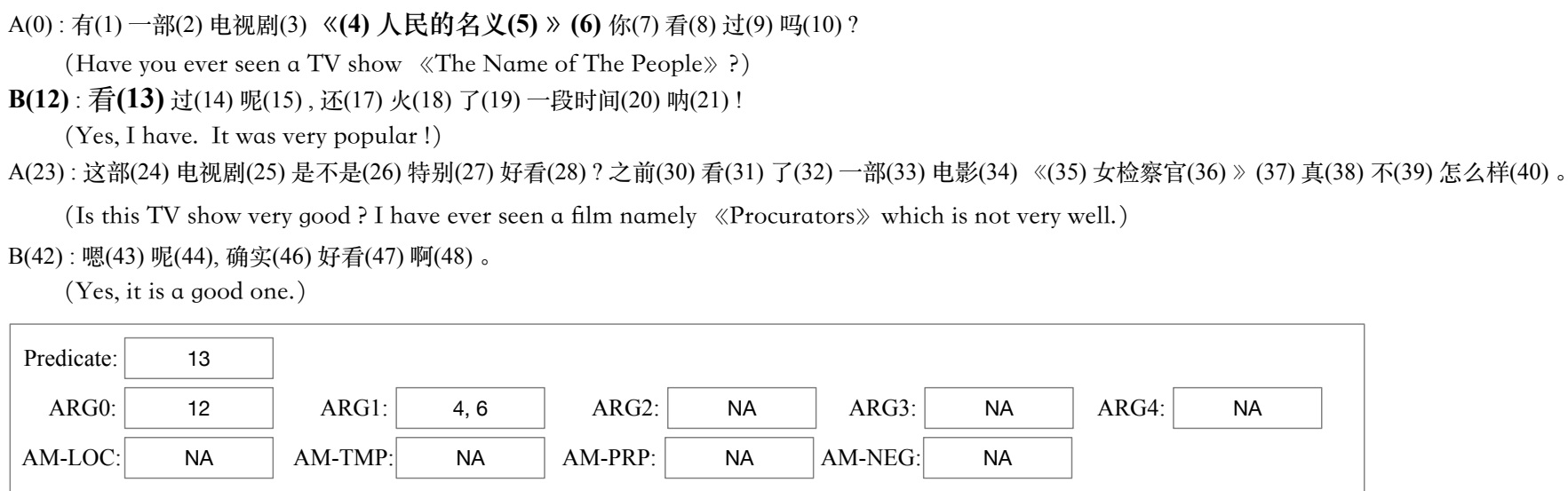

Fig. 2: Interface for the annotation.

\begin{tabular}{rcccccccc}
\hline Dataset & ARG0 & ARG1 & ARG2 & ARG3 & ARG4 & AM-TMP & AM-LOC & AM-PRP \\
\hline DuConv & $42.1(22.9)$ & $40.2(16.9)$ & $10.1(30.2)$ & $3.0(24.8)$ & $0.3(41.4)$ & $3.2(0.3)$ & $1.0(2.1)$ & $0.1(4.0)$ \\
PersonalDialog & $41.4(15.1)$ & $40.8(8.0)$ & $9.6(14.5)$ & $1.4(22.9)$ & $0.1(50.0)$ & $5.4(1.1)$ & $0.9(3.2)$ & $0.3(0.0)$ \\
NewsDialog & $42.2(26.8)$ & $41.7(16.3)$ & $7.3(20.5)$ & $1.4(31.1)$ & $0.1(83.3)$ & $6.1(0.2)$ & $0.9(0.0)$ & $0.3(0.0)$ \\
\hline
\end{tabular}

TABLE I: Percent $(\%)$ of each type of argument and its cross-turn ratio (shown inside parenthesis).

a Weibo message, other users may respond with comments that may receive further comments. This results in a tree structure rooted in the original Weibo post. An original post and one branch of its comments between the same user pairs are regarded as a dialogue session.

c) NewsDialog: This in-house dataset is collected in a similar way as [3] by asking two participants to discuss each provided document. It also follows the setting for constructing general open-domain dialogues: two participants engage in chitchat, and during the conversation, the topic is allowed to change naturally. Different from [3], who use Wikipedia articles, this dataset is supported by news, i.e., the participants have access to an information retrieval system that shows the news relevant to the conversation.

\section{B. Semantic Roles}

We follow PropBank [18], the most widely used standard for annotating predicate-argument structures. It has 32 standard semantic roles. By analyzing the conversation dataset, we adopt 9 core semantic roles in our dialogue SRL:

- Numbered arguments (ARG0-ARG4): Arguments defining verb-specific roles. Their semantics depends on the verb and the verb usage in a sentence or verb sense. In general, ARG0 stands for the agent and ARG1 corresponds to the patient or theme of the proposition, and these two are the most frequent roles. Numbered arguments reflect either the arguments that are required for the valency of a predicate or if not required, those that occur with high frequency in actual usage.

- Adjuncts: General arguments that any verbs may take optionally. In PropBank, there are 13 types of adjuncts, while in our dataset we only consider the most frequent four types of adjuncts, i.e., AM-LOC, AM-TMP, AM-PRP and AM-NEG. Specifically, the locative modifiers $(A M-L O C)$ indicate where the action takes place. The temporal arguments (AM-TMP) show when an action takes place, such as 很快 (soon) or 马 上 (immediately). Note that, the adverbs of frequency (e.g., 偶 尔 (sometimes), 总是 (always)), adverbs of duration (e.g., 过 两天 (in two days)) and repetition (e.g., 又 (again)) are also labeled as AM-TMP. Purpose clauses ( $A M-P R P)$ are used to show the motivation for an action. Clauses beginning with 为了 (in order to) and 因为 (because) are canonical purpose clauses. AM-NEG is used for elements such as “没有' (not) and “绝不' (no longer).

\section{Annotation Details}

There are two main types of semantic roles: span-based [33], [34] and dependency-based [35]. The former involves the start and end boundaries for each component, and the latter only considers the headword in a dependency tree for each component. We follow the span-based form, which has been adopted by most previous works.

a) Preprocessing: For each dialogue session, we first convert it to a paragraph by concatenating each utterance in the dialogue history. To explicitly indicate each utterance's speakers, we insert the corresponding speaker token at the beginning of each utterance before the concatenation. We then use Stanford CoreNLP [12] for sentence segmentation, tokenizing, and POS-tagging. We identify verbs by POS tagging with heuristics to filter out auxiliary verbs.

b) Labeling instructions: We ask five annotators who are familiar with PropBank semantic roles to annotate these dialogue sessions. Following the span-based annotation standard, annotators label the index ranges for each predicate and its arguments. In contrast to the standard sentence-level SRL, conversational SRL aims to additionally address the ellipsis and anaphora problems, which frequently occurred in the dialogue scenario. To this end, the annotators are instructed that a valid annotation must satisfy the following criteria: (1) 


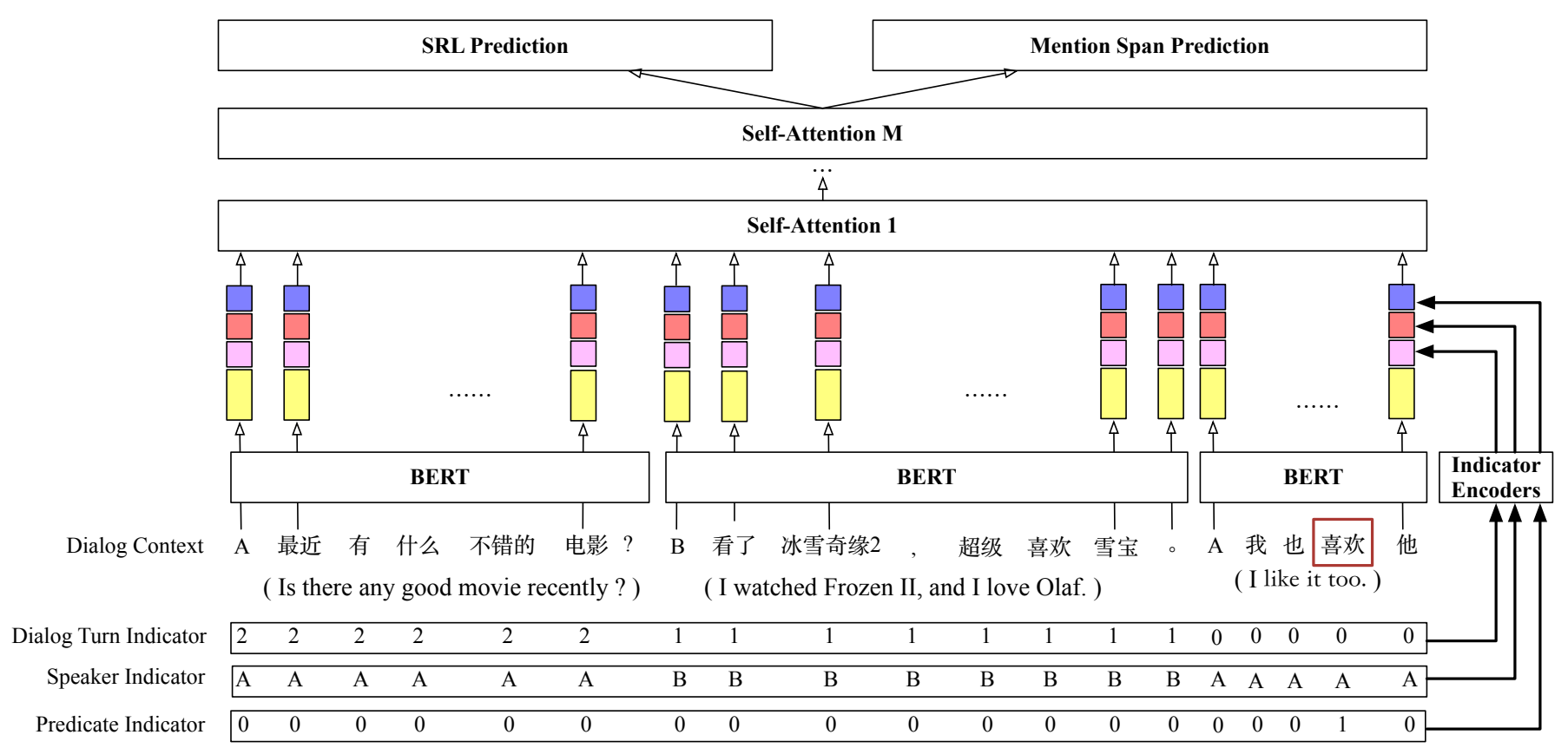

Fig. 3: Model architecture. It takes the sentences from Figure 1 as the inputs, where the associated predicate (“喜欢(like)”) is in bold font.

the argument should only appear in the current or previous turns; (2) the argument should not be assigned to a pronoun unless its reference could not be found in previous turns; (3) if the argument is the speaker or listener, it should be explicitly assigned to the special token we used to indicate the speaker (i.e., A or B). (4) in cases when there are multiple choices for labeling an argument, we select the one closest to the predicate. Figure 2 illustrates the annotation interface. The number in the bracket indicates the token index within the dialogue paragraph.

\section{Statistics}

We annotated 3,000 dialogue sessions from DuConv (33,673 predicates in 27,198 utterances), 300 sessions from PersonalDialog (1,441 predicates in 1,579 utterances) and 200 sessions from NewsDialog (3,621 predicates in 6,037 utterances). These datasets contain $21.89 \%, 12.56 \%$, and $20.01 \%$ arguments that are not in the same turn with their predicates. Also, $9.46 \%, 13.05 \%$, and $8.27 \%$ arguments are speaker arguments in these datasets, respectively. The average number of dialogue turns in three datasets are 9.0, 7.9 and 20.1, respectively. The average number of session tokens are 96.0, 100.1 and 230.1, respectively.

Table [I analyzes our datasets by listing the percent of each argument type and its cross-turn ratio. For all the three datasets, arguments ARGO, ARG1 and ARG2 count for the major proportion of the arguments. For adjunct-type arguments, AM-TMP and AM-LOC appear more than AM-PRP. It is likely because humans tend to avoid mentioning reasons for simplicity. Besides, the adjunct-type arguments have very low cross-turn ratios. This fits our intuition that humans usually mention the time and location when describing an event or a piece of news. For the following experiments, our annotations on DuConv are split into $80 \% / 10 \% / 10 \%$ as train/dev/test set, and the annotations on both PersonalDialog and NewsDialog serve only as test examples.

\section{TASK DEFINITION}

Formally, the input is a dialogue $C=\left(u_{1}, \ldots, u_{T}\right)$ of $T$ utterances, where $u_{t}=\left(w_{t, 1}, \ldots, w_{t,\left|u_{t}\right|}\right)$ consists of a sequence of words, and $u_{T}$ is the current turn. Following previous work [34], [36], we cast the task into a sequential labeling problem, where a label needs to be predicted for each token in both the current turn and the dialogue history, given a predicate. The label set draws from the cross of the standard BIO tagging scheme and the argument sets, and example labels are $B-A R G 1$ and I-ARG1 that represent the beginning and intermediate token for ARG1, respectively. We follow previous settings [34], [37], [38], where predicate positions are already marked in both training and evaluation sets, and only focus on predicting arguments.

\section{Model}

Our model adopts a state-of-the-art system [36] for conversations. As illustrated in Figure 3 it consists of an utterance encoder and $M$ self-attention layers. The input dialogue session is first fed to the utterance encoder to learn the contextual representations for each utterance. Then a stack of self-attention layers is further used to capture the dialog-level contextual representations. The topmost layer is the softmax classification layer.

a) Utterances Encoder: Given an input dialogue $C$, we first use a pre-trained language model, namely RoBERTa [39], to encode each utterance 3 This results in a series of word

\footnotetext{
3 https://github.com/brightmart/roberta_zh
} 
representations, which are supposed to capture the contextual information of words within utterances.

b) Indicator Embedding.: In addition to the utterance encoding, we also consider three types of indicator features closely related to our task. Conceptually, the first two indicators are mainly used to explicitly encode the speaker and dialogue turn information. Specifically, the first one is the speaker indicator capturing the speaker of each word. In our case, the speaker is either $A$ or $B^{4}$. The second type is the dialog-turn indicator, which calculates the relative distance in terms of dialogue turn between a word to the predicate one. The last one is the predicate indicator, which is typically used in the standard SRL to distinguish the predicate words from nonpredicate ones. Here, we mark the predicate word as 1 while other words are marked as 0 . These indicator embeddings are first concatenated to the contextual representation from the utterance encoder and then fed to the subsequent self-attention layers.

c) Self-Attention.: We use self-attention [40] to correlate the context of all dialogue histories. In particular, we first map the matrix of input vectors $\mathbf{H} \in \mathbb{R}^{N \times d}$ ( $N$ is the total number of words in $C ; d$ represents the mapped dimension) to queries $(\mathbf{Q})$, keys $(\mathbf{K})$ and values $(\mathbf{V})$ matrices by different linear projections:

$$
\left[\begin{array}{l}
\mathbf{K} \\
\mathbf{Q} \\
\mathbf{V}
\end{array}\right]=\left[\begin{array}{l}
\mathbf{W}_{k} \mathbf{H} \\
\mathbf{W}_{q} \mathbf{H} \\
\mathbf{W}_{v} \mathbf{H}
\end{array}\right]
$$

The attention weight is then computed by dot product between $\mathbf{Q}, \mathbf{K}$ and the selft-attention output $\mathbf{C} \in \mathbb{R}^{N \times d}$ is a weighted sum of values $\mathbf{V}$ :

$$
\mathbf{C}=\operatorname{Attention}(\mathbf{Q}, \mathbf{K}, \mathbf{V})=\operatorname{Softmax}\left(\frac{\mathbf{Q K}^{T}}{\sqrt{d_{k}}}\right) \mathbf{V}
$$

where $\mathbf{C}=\left(\mathbf{c}_{1}, \ldots, \mathbf{c}_{N}\right)$ are the word representations that capture both the utterance- and dialogue-level contextual information; $d_{k}$ represents the dimension of keys. Finally, predictions are made using a multi-layer perceptron with one hidden layer over the label set: $t_{i}=\operatorname{softmax}\left(\operatorname{MLP}\left(\boldsymbol{c}_{i}\right)\right)$.

d) Training: We train our model using two types of losses: SRL loss and mention span loss. Both two types of losses are conditionally independent and are calculated with different linear layers, given the deep features produced by our model, and the final loss for training is their combination.

Specifically, given a set of training instances, each containing a dialogue session $C=\left\{w_{1}, \ldots, w_{N}\right\}$, where $N$ represents the total number of words in the dialog, we train our model with a cross-entropy loss between the gold-standard labels $y$ $=\left\{y_{1}, y_{2}, \ldots, y_{N}\right\}$ and model distribution. In addition, we use the loss for predicting mention spans to better establish the argument boundaries:

$$
\mathcal{L}=-\frac{1}{N} \sum_{t=1}^{N} \underbrace{\log p\left(y_{t} \mid w_{t}, C ; \boldsymbol{\theta}\right)}_{\mathcal{L}_{S R L}}+\underbrace{\log p\left(z_{t} \mid w_{t}, C ; \boldsymbol{\theta}\right)}_{\mathcal{L}_{\text {span }}}
$$

where $\boldsymbol{\theta}$ represents the model parameters, and each $z_{t}$ is

\begin{tabular}{|c|c|}
\hline Utterance 1 & $\begin{array}{l}\text { 需要粤语 } \\
\text { (I may need Cantonese.) }\end{array}$ \\
\hline Utterance 2 & $\begin{array}{l}\text { 粤语ARG0是普通话ARG1 吗 } \\
\text { (Is Cantonese Mandarin ?) }\end{array}$ \\
\hline Utterance 3 & $\begin{array}{l}\text { 不算predicate 吧 } \\
\text { (Maybe Not.) }\end{array}$ \\
\hline Utterance $\mathbf{3}^{\prime}$ & $\begin{array}{l}\text { 粤语不算普通话吧 } \\
\text { (Cantonese may be not Mandarin.) }\end{array}$ \\
\hline
\end{tabular}
the gold mention-span label (also in BIO scheme) for $w_{t}$.

${ }^{4}$ The first speaker in a dialogue is viewed as speaker $A$.
TABLE II: One example of multi-turn dialogue. The goal of dialogue rewriting is to rewrite utterance 3 into $3^{\prime}$.

Since DuConv already provides the relevant KB fragment for dialogs, the mentions are generated by matching the $\mathrm{KB}$ entities with dialogue utterances.

\section{Applications}

In this paper, we investigate whether CSRL could benefit two major conversation-based tasks, i.e., multi-turn dialogue rewriter and dialogue generation. For each task, we first build a BERT-based baseline which already outperforms previous approaches, and then conduct experiments based on them. In this section, we first discuss the motivation of applying CSRL into these tasks before introducing how to incorporate CSRL features into baseline models.

\section{A. Multi-turn Dialogue ReWriting}

Recent work on dialogue generation [27]-[29] has achieved impressive progress for making single-turn responses, while producing coherent multi-turn replies still remains challenging. One important reason can be the long-range dependency issue caused by the lengthy context of the multi-turn scenario, where a generated reply needs to be coherent with every previous turn rather than only the last turn. To tackle this problem, the task of sentence rewriting was introduced to alleviate the longrange issue by merging relevant historical information into the latest turn, simplifying the original problem into a single-turn problem.

Conceptually, the current state-of-the-art rewriting models follow the conventional encoder-decoder architecture that first encodes the dialogue context into a distributional representation and then decodes it to the rewritten utterance. Their decoders mainly use global attention methods that attend to all words in the dialogue context without prior focus, which may result in inaccurate concentration on some dispensable words. This observation is expected since if the text is lengthy, it would be quite difficult for deep learning models to understand as it suffers from noise and pays vague attention to the text components.

Since CSRL could identify predicate-argument structures of a sentence, we believe that it can help pick out the important words that are semantically most related to the latest utterance that needs to be rewritten. For example, in Table II] our CSRL parser can find that the ARGO and ARG1 of “不算”(is not) are “粤语”(Cantonese) and “普通话”(Mandarin), respectively. Consequently, CSRL could guide the rewriter model to pay more attention to the semantically important words in the dialogue history, especially the omitted information that 


\begin{tabular}{|c|c|c|c|c|c|c|c|c|c|c|c|c|c|c|c|c|c|c|c|c|c|c|c|}
\hline \multirow{2}{*}{ Input } & \multicolumn{6}{|c|}{ Predicate-Argument Triples } & \multicolumn{12}{|c|}{ Dialog Context } & \multicolumn{5}{|c|}{ Rewrite } \\
\hline & 不 & 爱 & ARG1 & 苏 & 牙 & [EOS] & \begin{tabular}{|l|} 
\\
\end{tabular} & \begin{tabular}{|l|} 
爱 \\
\end{tabular} & 梅 & 西 & [EOS] & 苏 & \begin{tabular}{|l|} 
牙 \\
\end{tabular} & \begin{tabular}{|l|} 
呢 \\
\end{tabular} & [EOS] & 不 & 爱 & [EOS] & {$[\mathrm{BOS}]$} & 不 & \begin{tabular}{|l|} 
爱 \\
\end{tabular} & 苏 & 牙 \\
\hline $\begin{array}{c}\text { Token } \\
\text { Embeddings }\end{array}$ & E不 & E舜 & $\mathrm{E}_{\mathrm{ARG1}}$ & E苏 & E牙 & EEOS & E我 & E爱 & E棜 & $\mathrm{E}_{\text {西 }}$ & Eвоs & E勱 & E牙 & 巨呢 & Eés & E不 & E爱 & Eeos & Евоs & $\mathrm{E}_{\text {不 }}$ & E爱 & E水 & E稪 \\
\hline $\begin{array}{l}\text { Segment Type } \\
\text { Embeddings }\end{array}$ & & & E & & & & & & E & & & & & $\mathrm{E}_{\text {в }}$ & & & $\mathrm{E}_{A}$ & & & & $\mathrm{E}_{\mathrm{A}}$ & & \\
\hline $\begin{array}{l}\text { Position } \\
\text { Embeddings }\end{array}$ & $E_{0}$ & $E_{1}$ & $E_{2}$ & $E_{3}$ & $E_{4}$ & $E_{5}$ & $\mathrm{E}_{0}$ & $\mathrm{E}_{1}$ & $E_{2}$ & $\mathrm{E}_{3}$ & $\mathrm{E}_{4}$ & $\mathrm{E}_{0}$ & $\mathrm{E}_{1}$ & $\mathrm{E}_{2}$ & $\mathrm{E}_{3}$ & $\mathrm{E}_{0}$ & $E_{1}$ & $\mathrm{E}_{2}$ & $\mathrm{E}_{0}$ & $E_{1}$ & $\mathrm{E}_{2}$ & $\mathrm{E}_{3}$ & $\mathrm{E}_{4}$ \\
\hline
\end{tabular}

Fig. 4: The input representation of the rewriter and dialogue model.

appears in previous turns. Motivated by this observation, we attempt to apply CSRL into this task by first taking the CSRL parser to recognize the predicate-argument structures from dialog contexts and encode those structural information into the rewriter model.

Problem Formulation. Formally, an input for dialogue rewriting is a dialogue session $c=\left(u_{1}, \ldots, u_{N}\right)$ of $N$ utterances, where $u_{N}$ is the most recent utterance that needs to be revised. The output is $r$, the resulting utterance after recovering all coreference and omitted information in $u_{N}$. Our goal is to learn a model that can automatically rewrite $u_{N}$ based on the dialogue context.

ReWriter Model. Given a dialogue context $c$, we first apply an SRL parser to identify the predicate-argument structures $z$; then conditioned on $c$ and $z$, the rewritten utterance is generated as $p(r \mid c, z)$. The backbone of our infrastructure is similar to the transformer blocks in [41], which supports both bi-directional encoding and uni-directional decoding flexibly via specific self-attention masks. Specifically, we concatenate $z, c$ and $r$ as a sequence, feeding them into our model for training; during decoding, our model takes the $z$ and $c$ before generating the rewritten utterance word by word. Our model uses a pre-trained Chinese RoBERTa [39] for rich features.

Input Representation. For each token, its input representation is obtained by summing the embeddings for word, semantic role and position. One example is shown in Figure 4 and details are described in the following:

- The input is the concatenation of PA structures, dialog context, and rewritten utterance. Note that a PA structure is essentially in a tree format, where the root is a predicate and its children are corresponding semantic arguments. For the linearization, we decompose each PA structure into several triples of the form $<$ predicate, role, argument $>$ and concatenate them in a random order. A special end-of-utterance token (i.e., [EOS]) is appended to the end of each utterance for separation. Another begin-of-utterance token (i.e., [BOS]) is also added at the beginning of the rewritten utterance. The final hidden state of the last token in the final layer is used to predict the next token during generation.

- We expand the segment-type embeddings of BERT to distinguish different types of tokens. In particular, the type embedding $\mathrm{E}_{A}$ is added for the rewritten utterance, as well as dialogue utterances generated by the same speaker in the context; the type embedding $\mathrm{E}_{B}$ is used for the other speaker; $\mathrm{E}_{S R L}$ is used as the type embedding of the tokens in predicate-argument triples. Position embeddings are added according to the token position in each utterance. The input embedding is the summation of word embedding, segment embedding, and position embedding.

Attention Mask. Similar to TransferTransfo [42], we apply a future mask on the rewritten sequence; that is, the tokens in the rewritten utterance only attend on previous tokens in self-attention layers. Recall that we linearize a PA structure into a concatenation sequence of triples. Since these triples are randomly ordered, it may inevitably introduce noisy information when using a sequence encoder. To better reflect its structural information, we elaborate the attention mask on PA sequence: the tokens in the same PA triple have bidirectional attentions while tokens in different PA triples can not attend to each other. And the position embeddings of tokens in the PA sequence are added according to their positions in each distinct triple rather than the total PA sequence. In experiments, we find using these two designs helps our model to more efficiently use the SRL information.

Training We employ the NLL loss to train our model:

$$
\mathcal{L}=-\sum_{t=1}^{T} \log p\left(r_{t} \mid c, z, r_{<t} ; \boldsymbol{\theta}\right)
$$

where $\boldsymbol{\theta}$ represents the model parameters, $T$ is the length of the target response $r$, and $r_{<t}$ denotes previously generated words.

\section{B. Multi-turn Dialogue Response Generation}

In addition to multi-turn dialogue rewriting, we also conduct a preliminary study on multi-turn dialogue response generation, one of the main challenges in dialogue community. In contrast to the single-turn dialogue response generation, the ellipsis and anaphora may more frequently occur in multi-turn dialogues. However, previous approaches simply concatenate each multi-turn dialogue session as a "long" input utterance or adopt a hierarchical neural structure without analyzing the semantic information among these utterances. Intuitively, 


\begin{tabular}{|c|c|c|c|c|c|c|c|c|c|}
\hline \multirow{2}{*}{ Method } & \multicolumn{3}{|c|}{ DuConv } & \multicolumn{3}{|c|}{ PersonalDialog } & \multicolumn{3}{|c|}{ NewsDialog } \\
\hline & $\mathrm{F} 1_{a l l}$ & $\overline{\mathrm{F}} 1_{\text {cross }}$ & $\mathrm{F} 1_{i n t r a}$ & $\mathrm{~F} 1_{\text {all }}$ & $\overline{\mathrm{F}} 1_{\text {cross }}$ & $\mathrm{F} 1_{i n t r a}$ & $\overline{\mathrm{F}} 1_{\text {all }}$ & $\overline{\mathrm{F} 1_{\text {cross }}}$ & $\mathrm{F}_{\text {intra }}$ \\
\hline SRL-single & 42.8 & 0.0 & 51.2 & 44.9 & 0.0 & 50.8 & 54.0 & 0.0 & 61.6 \\
\hline SRL-entire & 42.7 & 1.1 & 51.2 & 42.5 & 1.8 & 49.0 & 52.5 & 1.0 & 60.2 \\
\hline SRL-rewrite & 39.5 & 3.8 & 49.2 & 37.5 & 0.5 & 44.9 & 44.0 & 2.0 & 54.6 \\
\hline SRL-coref & 40.9 & 8.1 & 49.4 & 40.0 & 1.1 & 46.5 & 45.1 & 4.2 & 54.3 \\
\hline SRL-DuConv-entire & 48.1 & 1.2 & 58.0 & 40.6 & 1.2 & 42.3 & 50.3 & 0.5 & 57.8 \\
\hline CSRL & 85.7 & 78.3 & 86.9 & 67.4 & 32.0 & 70.8 & 74.0 & 46.2 & 78.0 \\
\hline w/ pretrain & 85.9 & 78.5 & 87.0 & 68.7 & 34.5 & 71.9 & 74.7 & 48.4 & 78.3 \\
\hline w/o span loss & 85.4 & 78.0 & 86.6 & 66.8 & 31.2 & 68.3 & 72.9 & 46.0 & 76.6 \\
\hline
\end{tabular}

TABLE III: Evaluation results on the DuConv, PersonalDialog and NewsDialog datasets.

CSRL could naturally extract the semantic information which could help the model to better understand the dialogue context.

In this paper, we study the effect of CSRL by simply providing it as part of the input. Specifically, given a dialogue context $c$, we first apply our CSRL parser to identify the predicate-argument structures $z$; then conditioned on $c$ and $z$, the response is generated as $p(r \mid c, z)$. The backbone of our infrastructure is similar to the transformer blocks in [41], which supports both bi-directional encoding and unidirectional decoding flexibly via specific self-attention masks. Specifically, we concatenate $z, c$ and $r$ as a sequence, feeding them into our model for training; during decoding, our model takes the $z$ and $c$ before generating the response word by word. Our model uses a pre-trained Chinese RoBERTa [39] for rich features.

Input Representation. As shown in Figure 4, the input representation of each token is obtained by summing the embeddings for word, semantic role and position.

Attention Mask. Similar to the rewriter model, the tokens in the response only attend to previous tokens in self-attention layers; we adopt the elaborated attention mask design for the tokens in PA structures.

Training. We employ the same NLL loss as the rewriter model to train the dialogue model.

\section{EXPERIMENTS}

We conduct three types of experiments to evaluate the usefulness of CSRL. Specifically, the first experiment evaluates the performance of the CSRL parser, while the remaining two experiments investigate the performance improvement on the dialogue rewriting and generation task that could be achieved by introducing the CSRL information.

\section{A. CSRL Evaluation}

Baselines. We compare our model with baselines that explore major solutions to extend standard SRL on dialogues. All baselines have the same neural architecture and are trained with the same loss as our model. The only difference is that they are trained on the CoNLL-2012 benchmark [17], which contains roughly 117,089 standard SRL instances. More details are listed below:
- SRL-single/-entire: After training, both models are directly applied on our three test sets for evaluation. SRL-single just feeds each dialogue turn to the model without considering the dialogue history, and SRL-entire concatenates the dialogue history and current turn as a single utterance by replacing all intermediate periods with commas.

- SRL-rewrite: This method first employs a pre-trained conversation-based ReWriter model [15] to refine dialogue utterances so that the omitted information that occurred in previous turns could be retained in the resulting utterances. Then we use the same standard-SRL model to predict predicate-argument structures.

- SRL-coref: This method first uses the same standard SRL model to predict predicate-argument structures and then applies a state-of-the-art model [43] for coreference resolution to find the original mention for each argument.

- SRL-DuConv-entire: This model is mostly identical with SRL-entire, except that it is trained only with the instances where every predicate-argument structure is in the same sentence. It is introduced for examining the potential annotation bias that could favor our model.

Experimental Settings. We take the following settings for our models and baselines. We train our model (CSRL) on the training portion of DuConv, containing roughly 21, 600 instances. The dimensions of the predicate, speaker, and dialogturn indicator embeddings are 10,50 , and 50 , respectively. The hidden size $d$ of the self-attention layer is set to 1024 , and the number of heads is set to 8 . We use four self-attention layers and tune all the hyper-parameters on the development set.

Following the previous research of standard SRL, we evaluate our system on the micro-averaged F1 over the (predicate, argument, label) tuples. However, unlike sentence-level SRL systems, which only calculate F1 over all arguments (i.e., $\mathrm{F} 1_{\text {all }}$ ), we also calculate $\mathrm{F} 1$ over those arguments in the same, and different dialogue turns as predicates, namely $\mathrm{F} 1_{\text {intra }}$ and $\mathrm{F} 1_{\text {cross. }}$. We refer these two types of arguments as intra- and cross-arguments, respectively. For the SRL-rewrite baseline, an argument is considered as a cross-argument if it only appears in the rewritten sentence, not in the original one (before rewriting).

Main Results. Table IIII summarizes the results of all compared models on the DuConv, PersonalDialog and NewsDialog datasets. We can see that our model outperforms all the baselines by large margins even for the two out-of-domain 


\begin{tabular}{lcccccccc}
\hline Method & ARG0 & ARG1 & ARG2 & ARG3 & ARG4 & AM-TMP & AM-LOC & AM-PRP \\
\hline SRL-single & 43.4 & 49.1 & 2.6 & 0.0 & 0.0 & 74.9 & 30.4 & 50.5 \\
SRL-entire & 43.1 & 48.7 & 3.2 & 0.0 & 0.0 & 73.6 & 29.6 & 43.1 \\
\hline CSRL w/ pretrain & 90.1 & 85.4 & 75.4 & 72.8 & 46.4 & 82.9 & 79.2 & 34.4 \\
\hline
\end{tabular}

TABLE IV: Detailed scores on the test set of DuConv dataset.

\begin{tabular}{|c|c|}
\hline A: & 很快(soon) 到(is) 我们的(our) 结婚(wedding) 纪念日 (anniversary)了, 我们(we) 准备(prepare) 出去(go for) 旅游(travel)! \\
\hline B: & 带着(with) 孩子(kid) 旅游(travel) 是不是(whether or not) 要带(bring) 很多(many) 东西(stuff)啊！ \\
\hline A: & $\begin{array}{l}\text { 是啊(yes), 我们(we) 专门(specially) 买了(bought) 一个(a) 大箱子(suitcase), 不过(but) 还是(are still) 太多了(too } \\
\text { many), 根本(at all) 放不下(cannot fit)。 }\end{array}$ \\
\hline $\begin{array}{l}\text { Predicate } \\
\text { 还是 }\end{array}$ & $\begin{array}{l}\text { Gold and Predicted Arguments } \\
(A R G 0, \text { 东西), (ARG1, 太多了) }|| \mid \text { ( } A R G 0 \text {, 箱子), (ARG1, 太多了) }\end{array}$ \\
\hline
\end{tabular}

TABLE V: Example dialogues and predicted argument structures by our model.

datasets, where the performance gaps are 22.5 and 20 absolute F1 points, respectively. Although the standard CoNLL-2012 benchmark has many more instances, our comparisons suggest that these corpora do not provide sufficient knowledge for the dialogue scenario, where arguments usually occur in different utterances that can be far from their predicates. Training only with the single-sentence annotations of DuConv, SRLDuConv-entire is better than SRL-entire on DuConv test set, but it is much worse on the other test sets. The reason can be that SRL-DuConv-entire benefits from the in-domain advantage on DuConv, but it suffers from less training data on the other two test sets. This also indicates that the improvements of CSRL are not due to any potential annotation bias.

In addition to the standard SRL setting (SRL-single), we also investigate other potential solutions for tackling conversations. In contrast to SRL-single, which only uses the singleturn information, SRL-entire incorporates the whole dialogue history for sequence annotation. Intuitively, considering more context could allow the model to better find cross-arguments. However, we do not observe SRL-entire achieves better performance on these datasets. In fact, the contextual information can even hurt $\mathrm{F} 1_{\text {intra }}$ since those contextual information may introduce the noise in detecting intra-arguments.

Introducing a state-of-the-art rewrite component, i.e., SRLrewrite, can slightly improve the numbers on $\mathrm{F} 1_{\text {cross }}$, but it significantly hurts $\mathrm{F} 1_{\text {intra }}$, resulting in much worse performances on $F 1_{\text {all }}$. This observation conflicts with our intuition; that is, the rewrite model could recover all co-referred and omitted mentions by rewriting the current utterance. By analyzing 300 rewritten DuConv utterances by the rewrite model, we find that although the rewrite model can recover a few omitted mentions, the grammar of rewritten utterance is usually problematic. As a result, the standard SRL model trained on the normal corpus may fail in such noisy sentences.

Leveraging a state-of-the-art model [43] to generate coreference chains, SRL-coref gets an absolute $2.4 \%$ improvement on average over SRL-rewrite for the cross arguments, and SRLcoref is also slightly better than SRL-rewrite for the intra cases. However, it is still much worse than SRL-single and SRL-entire. After an investigation, we find that it is mostly due to the errors of coreference resolution, i.e., some correct arguments are erroneously attached to a coreference chain.
Discussion. We also investigate the impact of indicator embeddings on the performance. Experimental results show that without the dialog-turn encoding, the $\mathrm{F} 1_{\text {all }}$ performance of our model decreases to 82.8, 63.2, and 70.0 on DuConv, PersonalDialog, and NewsDialog, respectively. In particular, we find that removing the dialog-turn feature severely hurts the performance on cross-arguments. This result is expected since the dialog-turn feature essentially reduces the distance of words in different turns, which allows the model to more easily find the arguments that occurred in previous dialogue turns. Recall that, in the conversational SRL, the underlying speaker itself could also be a potential argument. Integrating the speaker feature is also observed to improve the performance on such arguments.

One may raise a natural question on whether existing standard SRL benchmarks are helpful for further improvement for our model, especially on $\mathrm{F}_{1}$ intra . To investigate this, we first pre-train our model on the training set of CoNLL 2012 (117, 089 examples) and fine-tune it on our annotated DuConv training set. From Table III. we can see that our model can benefit from the pre-training (CSRL $w /$ pretrain vs CSRL), achieving better performance on all datasets.

We additionally investigate the impact of span loss to CSRL performance. From Table III] we can see that without the span loss, the performance of CSRL consistently drops on three datasets. These results are expected since minimizing the span loss could encourage the model to better detect the argument boundaries.

We list the detailed performance of our best model in Table IV] The results of the two most competitive baselines are also shown for comparison. We can see that our model significantly outperforms these baselines on all semantic roles except for AM-PRP, which may be due to its low crossturn ratio. Besides, the lack of sufficient training data may also contribute to this situation. In particular, we find that the CoNLL 2012 contains 1,940 AM-PRP arguments, while our annotation only includes 75 appearances. We also observe similar situations where SRL-based baselines do relatively well for AM-TMP and AM-LOC, and one likely reason is that they also have a meager cross-turn ratio. Combining these observations, we may conclude that explicitly modeling conversational SRL is essential, because the ability for cross- 
turn reasoning cannot be acquired by training on sentence-level benchmarks.

Error Analysis. We analyze the errors of our model and find that most errors are caused by incorrect boundary detection and incorrect argument selection. In particular, the boundary detection errors mainly occurred in the entity mentions, while the lack of commonsense knowledge mainly causes argument selection errors. Table $\mathrm{V}$ gives an example of argument selection error. We can see that the correct $A R G 0$ of predicate “还是(are still)” is “东西(stuff)”, while our model predicts it as “箱子(suitcase)". To correctly find the ARG0 for this case, one should be aware of a suitcase is a container, and it has a capacity which can only carry limited stuff. We leave incorporating such commonsense knowledge for future work.

\section{B. Multi-turn Dialogue ReWriter Evaluation}

We evaluate our enhanced ReWriter model on a rewrite dataset built by [44]. This dataset is generated by crawling multi-turn conversational data from several popular Chinese social media platforms. Specifically, this dataset contains 17,890 examples, which are further split as $80 \% / 10 \% / 10 \%$ for training/development/testing, respectively. The hyperparameters used in our model are listed as follows. The network parameters of our model are initialized using RoBERTa. The batch size is set to 32. We use Adam [45] with learning rate 5 e-5 to update parameters. We directly employ the CSRL parser that pre-trained on the DuConv to conduct the experiments on the rewriting task.

Results. Following previous works, we used BLEU, ROUGE, and the exact match score (EM) (the percentage of decoded sequences that exactly match the human references). We implemented three baselines that use the same transformer-based encoder but differ in the choice of the decoder. Specifically, Trans-Gen uses a pure generation decoder which generates words from a fixed vocabulary; Trans-Pointer applies a pure pointer-based decoder [46] which can only copy the word from the input; Trans-Hybrid uses a hybrid pointer+generation decoder as in [47], which can either copy the words from the input or generate words from a fixed vocabulary. Table VI summarizes the results of our model and these baselines.

Discussion. We can see that even without the SRL information, our model still significantly outperforms these baselines, indicating that adopting a pre-trained language model could greatly improve the performance of such a generation task. We can also see that the model with the pointer-based decoder achieves better performance than the generation-based and the hybrid one, which is similar to the observation as in [44]. This result is expected since there is a high chance the coreference or omission could be perfectly resolved by only using previous dialogue turns. In addition, we find that incorporating the SRL information can further improve the performance by at 1.45 BLEU-1 and 1.6 BLEU-2 points, achieving state-of-the-art performances.

Let us first look at the impact of attention mask design on our model. To incorporate the CSRL information into our model, we view the linearized predicate-argument structures as a regular utterance (say $u_{p a}$ ) and append it in the front of the input. We experimented with two choices of attention masks. Specifically, the first one is a bidirectional mask (referred as Bi-mask), that is, words in $u_{p a}$ could attend each other; the second one (referred as Triple-mask) only allows words to attend its neighbors in the same triple, i.e., words in different triples are not visible to each other. From Table VI we can see that the latter one is significantly better than the first one. We think the main reason is that the second design independently encodes each predicate-argument triple, which prevents the unnecessary triple-internal attentions, better mimicking the SRL structures.

Since our framework works in a pipeline fashion, one bottleneck of our system can lie in the performance of the CSRL parser. One natural question is how accurate our CSRL parser can be and how much performance improvement for the rewriter model we could have by introducing the CSRL information. To investigate this, we employ a standard SRL parser ${ }^{5}$ to analyze the gold rewritten utterance. These extracted PA structures are considered as the upper bound to measure the accuracy of our CSRL parser. In particular, we evaluate our CSRL parser on the micro-averaged F1 over the (predicate, argument, label) tuples. We find our CSRL parser achieves 75.66 precision, 74.47 recall, and $75.06 \mathrm{~F}_{1}$. On the other hand, we use the upper bound SRL results instead of our CSRL parsing results to train and test the model (referred as BERT+CSRL (upper bound)). From Table VI, we can see that all evaluation scores are significantly improved. This result indicates that the performance of our rewriter model is highly relevant to the CSRL parser, and the performance of our current CSRL parser is still far from satisfactory, which we leave for future work.

We also investigate which type of dialogues our model could benefit from incorporating CSRL information? By analyzing the dialogues and our predicted rewritten utterances, we find that the CSRL information mainly improves the performance on the dialogues that require information completion. One omitted information is considered as properly completed if the rewritten utterance recovers the omitted words. We find the CSRL parser naturally offers important guidance into the selection of omitted words.

Recall that, there is one additional scope option to apply the CSRL parser to extract PA structures, i.e., only working on the last utterance that needs to be rewritten. We evaluate this option on our dataset (referred as BERT+Partial-CSRL) and the results are shown in Table $\mathrm{VI}$. We can see that reducing the CSRL scope may slightly hurt the performance, which we think is because larger CSRL scope could provide additional guidance for the rewriter model.

\section{Multi-turn Dialogue Generation Evaluation}

We evaluate our dialogue response generation model on the DuConv dataset, which includes 19,858/2,000/5,000 dialogue sessions for training/developing/testing. Recall that, DuConv requires the dialogue model to generate the response using the given knowledge graph. We simply linearize the given knowledge graph and append it to the dialogue history, which

\footnotetext{
${ }^{5}$ This parser is trained on the CoNLL-2012 dataset.
} 


\begin{tabular}{lccccccc}
\hline & BLEU-1 & BLEU-2 & BLEU-4 & ROUGE-1 & ROUGE-2 & ROUGE-L & EM \\
\hline Trans-Gen & 78.18 & 70.31 & 51.85 & 83.1 & 67.84 & 81.98 & 24.12 \\
Trans-Pointer & 83.22 & 78.32 & 64.08 & 87.89 & 77.94 & 86.88 & 36.54 \\
Trans-Hybrid & 82.92 & 77.65 & 62.54 & 87.59 & 76.91 & 86.66 & 35.03 \\
Su et al. [44] & 85.41 & 81.67 & 70.00 & 89.75 & 81.84 & 88.56 & 46.33 \\
\hline BERT & 88.21 & 85.17 & 75.64 & 90.73 & 84.35 & 89.47 & 57.36 \\
BERT + CSRL & & & & & & & \\
$\quad$ w/ Bi-mask & 88.89 & 85.88 & 76.36 & 90.92 & 85.00 & 89.72 & 58.36 \\
$\quad$ w/ Triple-mask & $\mathbf{8 9 . 6 6}$ & $\mathbf{8 6 . 7 8}$ & $\mathbf{7 7 . 7 6}$ & $\mathbf{9 1 . 8 2}$ & $\mathbf{8 5 . 8 7}$ & $\mathbf{9 0 . 5 2}$ & $\mathbf{6 0 . 4 9}$ \\
BERT + Partial-CSRL & 89.46 & 86.57 & 77.75 & 91.60 & 85.60 & 90.50 & 59.15 \\
\hline BERT + CSRL (upper bound) & 93.34 & 91.38 & 84.97 & 94.94 & 90.45 & 93.86 & 71.96 \\
\hline
\end{tabular}

TABLE VI: Evaluation results on the rewriting task.

\begin{tabular}{lccc}
\hline Method & BLEU 1/2 & DISTINCT 1/2 & Human \\
\hline Wu et al. [4] & $0.347 / 0.198$ & $0.057 / 0.155$ & 2.62 \\
Ours & $\mathbf{0 . 4 2 1 / 0 . 2 8 6}$ & $\mathbf{0 . 1 1 3 / 0 . 3 1 2}$ & $\mathbf{3 . 6 1}$ \\
$\quad$ w/o CSRL & $0.395 / 0.268$ & $0.103 / 0.208$ & 3.27 \\
\hline
\end{tabular}

TABLE VII: Evaluation results of dialogue response generation models on DuConv.

is viewed as the dialogue context. To evaluate the performance of the dialogue model, we leverage several common metrics, including BLEU and DISTINCT1/2. Table VII lists the results of our model and previous approaches on DuConv.

We can see that even without the CSRL information, our BERT-based baseline could already outperform the previous approach in terms of BLEU scores. After introducing the predicate-argument information, the performance could be significantly improved. In addition to the automatic evaluation criteria, we also perform human evaluation on the generation results. Specifically, we randomly select 400 generation results and employ three annotators to evaluate the coherence of the response against the dialogue context by giving a score range from 1 (worst) to 5 (best). Experimental results show that in around $75 \%$ of cases, CSRL could improve the coherence of the generated results. In experiments, we also find the attention mask design is critical to achieving such improvement; that is, the triple-mask is significantly better than the bidirectional mask.

\section{CONCLUSIONS AND Future WORK}

Standard SRL models trained on benchmarks of sentencelevel annotations may fail to analyze multi-turn dialogues where ellipsis and anaphora frequently occur. We introduced the task of conversational SRL (CSRL), where arguments can be dialogue participants or spans in the dialogue context. To facilitate training and evaluation of data-driven methods, we collected three high-quality, manually annotated datasets. We also designed a BERT-based model to compare CSRL with standard SRL on parsing out-of-domain dialogues. Experimental results show that our model trained by CSRL not only significantly outperforms standard-SRL-based baselines but also achieves satisfactory robustness on domain transfer. Further explorations of CSRL on dialogue response generation and dialogue context rewriting also demonstrate superior performances than previous state-of-the-art methods.
In the future, we will explore more applications of the CSRL in the dialogue community and improve the robustness of the CSRL on various domains.

\section{REFERENCES}

[1] Y. Li, H. Su, X. Shen, W. Li, Z. Cao, and S. Niu, "Dailydialog: A manually labelled multi-turn dialogue dataset," in Proceedings of the Eighth International Joint Conference on Natural Language Processing (Volume 1: Long Papers), 2017, pp. 986-995.

[2] S. Zhang, E. Dinan, J. Urbanek, A. Szlam, D. Kiela, and J. Weston, "Personalizing dialogue agents: I have a dog, do you have pets too?" in Proceedings of the 56th Annual Meeting of the Association for Computational Linguistics (Volume 1: Long Papers), 2018, pp. 22042213.

[3] E. Dinan, S. Roller, K. Shuster, A. Fan, M. Auli, and J. Weston, "Wizard of wikipedia: Knowledge-powered conversational agents," arXiv preprint arXiv:1811.01241, 2018.

[4] W. Wu, Z. Guo, X. Zhou, H. Wu, X. Zhang, R. Lian, and H. Wang, "Proactive human-machine conversation with explicit conversation goal," in Proceedings of the 57th Annual Meeting of the Association for Computational Linguistics, 2019, pp. 3794-3804.

[5] H. Zhou, C. Zheng, K. Huang, M. Huang, and X. Zhu, "KdConv: A Chinese multi-domain dialogue dataset towards multi-turn knowledgedriven conversation," in Proceedings of the 58th Annual Meeting of the Association for Computational Linguistics. Online: Association for Computational Linguistics, Jul. 2020, pp. 7098-7108. [Online]. Available: https://www.aclweb.org/anthology/2020.acl-main.635

[6] M. Henderson, B. Thomson, and J. D. Williams, "The second dialog state tracking challenge," in Proceedings of the 15th Annual Meeting of the Special Interest Group on Discourse and Dialogue (SIGDIAL). Philadelphia, PA, U.S.A.: Association for Computational Linguistics, Jun. 2014, pp. 263-272. [Online]. Available: https: //www.aclweb.org/anthology/W14-4337

[7] N. Mrkšić, D. O. Séaghdha, T.-H. Wen, B. Thomson, and S. Young, "Neural belief tracker: Data-driven dialogue state tracking," in Proceedings of the 55th Annual Meeting of the Association for Computational Linguistics (Volume 1: Long Papers), 2017, pp. 1777-1788.

[8] P. Budzianowski, T.-H. Wen, B.-H. Tseng, I. Casanueva, S. Ultes, O. Ramadan, and M. Gasic, "Multiwoz-a large-scale multi-domain wizardof-oz dataset for task-oriented dialogue modelling," in Proceedings of the 2018 Conference on Empirical Methods in Natural Language Processing, 2018, pp. 5016-5026.

[9] E. Choi, H. He, M. Iyyer, M. Yatskar, W.-t. Yih, Y. Choi, P. Liang, and L. Zettlemoyer, "Quac: Question answering in context," in Proceedings of the 2018 Conference on Empirical Methods in Natural Language Processing, 2018, pp. 2174-2184.

[10] S. Reddy, D. Chen, and C. D. Manning, "Coqa: A conversational question answering challenge," Transactions of the Association for Computational Linguistics, vol. 7, pp. 249-266, 2019.

[11] Y.-J. Kim, "Subject/object drop in the acquisition of korean: A crosslinguistic comparison," Journal of East Asian Linguistics, vol. 9, no. 4, pp. 325-351, 2000.

[12] C. D. Manning, M. Surdeanu, J. Bauer, J. Finkel, S. J. Bethard, and D. McClosky, "The Stanford CoreNLP natural language processing toolkit," in Association for Computational Linguistics (ACL) System Demonstrations, 2014, pp. 55-60. [Online]. Available: http://www. aclweb.org/anthology/P/P14/P14-5010 
[13] M. Gardner, J. Grus, M. Neumann, O. Tafjord, P. Dasigi, N. F. Liu, M. Peters, M. Schmitz, and L. Zettlemoyer, "Allennlp: A deep semantic natural language processing platform," in Proceedings of Workshop for NLP Open Source Software (NLP-OSS), 2018, pp. 1-6.

[14] A. Elgohary, D. Peskov, and J. Boyd-Graber, "Can you unpack that? learning to rewrite questions-in-context," in Proceedings of the 2019 Conference on Empirical Methods in Natural Language Processing and the 9th International Joint Conference on Natural Language Processing (EMNLP-IJCNLP), 2019, pp. 5917-5923.

[15] H. Su, X. Shen, R. Zhang, F. Sun, P. Hu, C. Niu, and J. Zhou, "Improving multi-turn dialogue modelling with utterance ReWriter," in Proceedings of the 57th Annual Meeting of the Association for Computational Linguistics, 2019, pp. 22-31.

[16] Y. Zheng, G. Chen, M. Huang, S. Liu, and X. Zhu, "Personalized dialogue generation with diversified traits," arXiv preprint arXiv:1901.09672, 2019.

[17] S. Pradhan, A. Moschitti, N. Xue, H. T. Ng, A. Björkelund, O. Uryupina, Y. Zhang, and Z. Zhong, "Towards robust linguistic analysis using ontonotes," in Proceedings of the Seventeenth Conference on Computational Natural Language Learning, 2013, pp. 143-152.

[18] X. Carreras and L. Màrquez, "Introduction to the CoNLL-2005 shared task: Semantic role labeling," in CoNLL-2005, Ann Arbor, Michigan, June 2005. [Online]. Available: http://www.aclweb.org/ anthology/W05-0620

[19] J. Ruppenhofer, C. Sporleder, R. Morante, C. Baker, and M. Palmer, "Semeval-2010 task 10: Linking events and their participants in discourse," in Proceedings of the Workshop on Semantic Evaluations. Recent Achievements and Future Directions, 2009, pp. 106-111.

[20] M. Gerber and J. Y. Chai, "Semantic role labeling of implicit arguments for nominal predicates," Computational Linguistics, vol. 38, no. 4, pp. 755-798, 2012.

[21] M. Roth and A. Frank, "Inducing implicit arguments from comparable texts: A framework and its applications," Computational Linguistics, vol. 41, no. 4, pp. 625-664, 2015.

[22] T.-H. Wen, D. Vandyke, N. Mrkšić, M. Gasic, L. M. R. Barahona, P.$\mathrm{H}$. Su, S. Ultes, and S. Young, "A network-based end-to-end trainable task-oriented dialogue system," in Proceedings of the 15th Conference of the European Chapter of the Association for Computational Linguistics: Volume 1, Long Papers, 2017, pp. 438-449.

[23] O. Vinyals and Q. Le, "A neural conversational model," in ICML Deep Learning Workshop, 2015.

[24] A. Sordoni, M. Galley, M. Auli, C. Brockett, Y. Ji, M. Mitchell, J.-Y Nie, J. Gao, and B. Dolan, "A neural network approach to contextsensitive generation of conversational responses," in Proceedings of the 2015 Conference of the North American Chapter of the Association for Computational Linguistics: Human Language Technologies, 2015, pp. 196-205.

[25] J. Li, M. Galley, C. Brockett, J. Gao, and B. Dolan, "A diversitypromoting objective function for neural conversation models," in Proceedings of the 2016 Conference of the North American Chapter of the Association for Computational Linguistics: Human Language Technologies, 2016, pp. 110-119.

[26] I. V. Serban, A. Sordoni, Y. Bengio, A. Courville, and J. Pineau, "Building end-to-end dialogue systems using generative hierarchical neural network models," in Thirtieth AAAI Conference on Artificial Intelligence, 2016.

[27] I. V. Serban, A. Sordoni, R. Lowe, L. Charlin, J. Pineau, A. Courville, and Y. Bengio, "A hierarchical latent variable encoder-decoder model for generating dialogues," in Thirty-First AAAI Conference on Artificial Intelligence, 2017.

[28] T. Zhao, R. Zhao, and M. Eskenazi, "Learning discourse-level diversity for neural dialog models using conditional variational autoencoders," in Proceedings of the 55th Annual Meeting of the Association for Computational Linguistics (Volume 1: Long Papers), 2017, pp. 654-664.

[29] Y. Shao, S. Gouws, D. Britz, A. Goldie, B. Strope, and R. Kurzweil, "Generating high-quality and informative conversation responses with sequence-to-sequence models," in Proceedings of the 2017 Conference on Empirical Methods in Natural Language Processing, 2017, pp. 2210 2219.

[30] C. Xing, W. Wu, Y. Wu, J. Liu, Y. Huang, M. Zhou, and W.-Y. $\mathrm{Ma}$, "Topic aware neural response generation," in Thirty-First AAAI Conference on Artificial Intelligence, 2017.

[31] Y. Zhang, X. Gao, S. Lee, C. Brockett, M. Galley, J. Gao, and B. Dolan, "Consistent dialogue generation with self-supervised feature learning," arXiv preprint arXiv:1903.05759, 2019.
[32] D. M. Blei, A. Y. Ng, and M. I. Jordan, "Latent dirichlet allocation," Journal of machine Learning research, vol. 3, no. Jan, pp. 993-1022, 2003.

[33] H. Ouchi, H. Shindo, and Y. Matsumoto, "A span selection model for semantic role labeling," arXiv preprint arXiv:1810.02245, 2018.

[34] Z. Tan, M. Wang, J. Xie, Y. Chen, and X. Shi, "Deep semantic role labeling with self-attention," in Thirty-Second AAAI Conference on Artificial Intelligence, 2018.

[35] Z. Li, S. He, H. Zhao, Y. Zhang, Z. Zhang, X. Zhou, and X. Zhou, "Dependency or span, end-to-end uniform semantic role labeling," in Proceedings of the AAAI Conference on Artificial Intelligence, vol. 33, 2019, pp. 6730-6737.

[36] P. Shi and J. Lin, "Simple bert models for relation extraction and semantic role labeling," arXiv preprint arXiv:1904.05255, 2019.

[37] L. He, K. Lee, M. Lewis, and L. Zettlemoyer, "Deep semantic role labeling: What works and what's next," in Proceedings of the 55th Annual Meeting of the Association for Computational Linguistics (Volume 1: Long Papers), 2017, pp. 473-483.

[38] D. Marcheggiani, J. Bastings, and I. Titov, "Exploiting semantics in neural machine translation with graph convolutional networks," in Proceedings of the 2018 Conference of the North American Chapter of the Association for Computational Linguistics: Human Language Technologies, Volume 2 (Short Papers), 2018, pp. 486-492.

[39] Y. Liu, M. Ott, N. Goyal, J. Du, M. Joshi, D. Chen, O. Levy, M. Lewis, L. Zettlemoyer, and V. Stoyanov, "Roberta: A robustly optimized bert pretraining approach," arXiv preprint arXiv:1907.11692, 2019.

[40] A. Vaswani, N. Shazeer, N. Parmar, J. Uszkoreit, L. Jones, A. N. Gomez, Ł. Kaiser, and I. Polosukhin, "Attention is all you need," in Advances in neural information processing systems, 2017, pp. 5998-6008.

[41] L. Dong, N. Yang, W. Wang, F. Wei, X. Liu, Y. Wang, J. Gao, M. Zhou, and H.-W. Hon, "Unified language model pre-training for natural language understanding and generation," in Advances in Neural Information Processing Systems, 2019, pp. 13042-13 054.

[42] T. Wolf, V. Sanh, J. Chaumond, and C. Delangue, "Transfertransfo: A transfer learning approach for neural network based conversational agents," arXiv preprint arXiv:1901.08149, 2019.

[43] M. Joshi, O. Levy, L. Zettlemoyer, and D. Weld, "BERT for coreference resolution: Baselines and analysis," in Proceedings of the 2019 Conference on Empirical Methods in Natural Language Processing and the 9th International Joint Conference on Natural Language Processing (EMNLP-IJCNLP), 2019, pp. 5803-5808.

[44] H. Su, X. Shen, R. Zhang, F. Sun, P. Hu, C. Niu, and J. Zhou, "Improving multi-turn dialogue modelling with utterance rewriter," arXiv preprint arXiv:1906.07004, 2019.

[45] D. P. Kingma and J. Ba, "Adam: A method for stochastic optimization," arXiv preprint arXiv:1412.6980, 2014.

[46] O. Vinyals, M. Fortunato, and N. Jaitly, "Pointer networks," in Advances in neural information processing systems, 2015, pp. 2692-2700.

[47] A. See, P. J. Liu, and C. D. Manning, "Get to the point: Summarization with pointer-generator networks," arXiv preprint arXiv:1704.04368, 2017. 